\title{
The Role of Kinesthetic Reference Frames in Two-Handed Input Performance
}

\author{
Ravin Balakrishnan ${ }^{1,2}$ \\ ${ }^{1}$ Alias|wavefront \\ 210 King Street East \\ Toronto, Ontario \\ Canada M5A 1J7 \\ ravin@aw.sgi.com
}

\author{
${ }^{2}$ Dept. of Computer Science \\ University of Toronto \\ Toronto, Ontario \\ Canada M5S 3G4 \\ ravin@dgp.toronto.edu
}

\author{
Ken Hinckley ${ }^{3}$ \\ ${ }^{3}$ Microsoft Research \\ One Microsoft Way \\ Redmond, WA \\ USA 98052 \\ kenh@microsoft.com
}

\begin{abstract}
We present experimental work which explores how the match (or mismatch) between the input space of the hands and the output space of a graphical display influences twohanded input performance. During interaction with computers, a direct correspondence between the input and output spaces is often lacking. Not only are the hands disjoint from the display space, but the reference frames of the hands may in fact be disjoint from one another if two separate input devices (e.g. two mice) are used for twohanded input. In general, we refer to the workspace and origin within which the hands operate as kinesthetic reference frames. Our goal is to better understand how an interface designer's choice of kinesthetic reference frames influences a user's ability to coordinate two-handed movements, and to explore how the answer to this question may depend on the availability of visual feedback. Understanding this issue has implications for the design of two-handed interaction techniques and input devices, as well as for the reference principle of Guiard's Kinematic Chain model of human bimanual action. Our results suggest that the Guiard reference principle is robust with respect to variances in the kinesthetic reference frames as long as appropriate visual feedback is present.
\end{abstract}

\section{Keywords}

two-handed input, kinesthesia, visual feedback, Guiard theory, input, interaction techniques,

\section{INTRODUCTION}

A number of systems have explored two-handed interaction techniques for desktop interaction [3][4][14], interactive 3D graphics [2][9], and virtual environments [17]. As a whole, these systems provide a number of case studies that demonstrate compelling two-handed input techniques. However, without a better understanding of how humans use two hands, there is little scientific knowledge to guide the design of new two-handed user interfaces. To better

\section{Published in UIST 1999, ACM Symposium on User Interface Software and Technology, CHI Letters, 1(1), p. 171-178. New York: ACM}

characterize the nature of two-handed interaction understanding what works well, what factors are important, and why - we need systematic experiments which explore and quantify human bimanual skills and thus pave the way for future designs.

Guiard's Kinematic Chain (KC) model [5] of human bimanual action is a fundamental theoretical insight that drives much of the current design and experimental research in two-handed interaction [2][9][12][14][15]. The $\mathrm{KC}$ model proposes general principles governing asymmetric bimanual action, which are two-handed manipulations where each hand plays a different role. The most important principle for our purposes is right-to-left spatial reference in manual motion (the "Guiard reference principle" hereafter). For right handers, this means that the right hand moves relative to the frame of reference defined by the left hand: e.g., when writing on a piece of paper, the left hand orients the page while the right hand moves the pen $^{1 !}$.

Previous experimental studies supporting the Guiard reference principle [5][6][10][12] have explored settings where there is a direct correspondence between visual feedback (the visible movements of the hands and the resulting ink on the page for the handwriting task, for example) and the user's kinesthetic sense of where one hand is relative to the other (via feedback produced within the user's own muscles and joints). In typical computer usage, however, such a direct correspondence between the input output spaces is often lacking. For example, when moving a mouse, one's hand moves in a space that is separate from the display. When using two mice, both mice map to the same output space but are manually operated in two separate input spaces (reference frames).

Although Guiard states his reference principle in general terms, we are not aware of any experiments which explore if, or how, the Guiard reference principle may apply to twohanded manipulation when the kinesthetic reference

\footnotetext{
${ }^{1}$ For left-handers, hand roles would be reversed. For ease of understanding, throughout this paper we use the terms left hand and right hand to denote a user's non-preferred and preferred hand respectively.
} 
frame(s) and visual feedback become separated in such a manner. Does the reference principle only apply directly to a unified combination of kinesthetic and visual feedback? Are there significant human two-handed performance bottlenecks which arise when, for example, the hands work in two separate kinesthetic reference frames, each with its own independent origin (as is the case when using two mice)?

This is not only a fundamental theoretical issue, but also has significant implications for the design and implementation of two-handed user interfaces and input devices, and thus is a problem which the interface design community needs to address. The experimental findings presented in this paper suggest that two-handed input performance, as well as the Guiard reference principle itself, are both robust with respect to variations in kinesthetic reference frames as long as appropriate visual feedback is present. However, if visual feedback is lacking or a high level of visual diversion is required for a task or interaction technique, emphasis must instead be given to a system in which the sensed positions of the input devices correspond closely to the physical separation between the two hands.

Note that our present experiment is not intended to validate the Guiard principle itself, as this issue has been explored by previous studies and analyses [5][6][10][12]. Our present study suggests that, under the assumption that the Guiard reference principle is indeed correct, the principle also applies regardless of whether the kinesthetic reference frames for each hand are unified by a common origin. Furthermore, visual reference in feedback on the screen alone is sufficient for Guiard's reference principle to apply. However, when visual feedback is absent, kinesthetic feedback in the form of body-relative cues are sufficient to guide two handed manipulation. In this sense neither visual nor kinesthetic feedback are individually essential to the Guiard reference principle, suggesting that the reference principle describes asymmetric two-handed manipulation in a very fundamental way which is apparently not directly dependent on either of these feedback modalities.

\section{PREVIOUS WORK}

Several experimental studies have quantified performance advantages for two-handed input techniques over traditional one-handed techniques. Buxton and Myers [4] showed that scrolling with touch-sensitive strips in the left hand could result in improved performance. Balakrishnan and Kurtenbach [2] explored bimanual camera control and object manipulation using mice in both the left and right hands. They found that a $3 \mathrm{D}$ target selection task was $20 \%$ faster with the two-handed technique. For a more complicated object docking task, performance advantages arose only after sufficient practice. Although both these studies show that two-handed techniques can be useful, they do not quantify potential factors that may drive twohanded performance itself.
Indeed, there are few examples of such studies. Guiard, in additional to his original work proposing the reference principle for asymmetric two-handed interaction, presents a tapping task with bimanually held rods which demonstrates an asymmetric division of labor between the hands [6]. Hinckley et. al. [10] present an experimental analysis of a bimanual pointing task which suggests that the Guiard reference principle is correct but that the task difficulty is also an important factor. This study used physical objects, however, meaning that there was a direct correspondence between visual and kinesthetic reference frames at all times.

A number of bimanual tasks have been studied in the psychology and motor behavior literatures, including hand lateralization [1][21], bimanual pointing to separate targets [13][16][24], and bimanual tapping of rhythms [18][25]. Preilowski [19][20] explores a two-handed steering task using hand cranks, each of which controls one degree-offreedom of a cursor. After practice, normal subjects can steer the cursor without visual feedback, whereas patients with damage to the anterior commissure cannot.

Other research suggests that humans have a keen kinesthetic sense of where their hands are relative to one another, which is independent of visual feedback [8]. One interpretation of this result is that the kinesthetic sense may supplement visual feedback and thus help users to naturally coordinate two-handed movements, although such a conclusion remains unproven. This study also did not look at how task performance interacts with varying kinesthetic reference frames when visual feedback is present.

Mine et. al. [17] present two-handed interaction techniques for immersive 3D virtual environments. An evaluation of the techniques quantifies improved performance for widgets held in one's hand, rather than floating in space. Another evaluation suggests that an offset (or variable offset) of a held object from the position of the user's hand, which essentially corresponds to varying the kinesthetic reference frames, results in significantly slower performance. To our knowledge, such issues have not been studied for two-handed input with 2D desktop systems.

Sellen et. al. [22] present experimental results which suggest that kinesthetic feedback is a more salient modality than visual feedback in preventing mode errors. This result demonstrates a situation in which kinesthetic feedback can be important, even when visual feedback is simultaneously available. However, it is not clear if kinesthetic feedback is a significant factor for two-handed input performance.

Visual dominance deals with the phenomena resulting from the tendency for vision to dominate other modalities. For example, several experiments suggest that if vision and kinesthesia are placed in conflict, subjects respond appropriately for the visual feedback [23]. Also, it has been shown that a combination of light and kinesthesia can lead to a response time slower than light alone, because visual 
attention slows down the more rapid processing of the kinesthetic stimulus [23].

\section{EXPERIMENT}

\section{Task}

We chose to use the colorized connect-the-dots task of Kabbash [12]. We feel this task is representative of asymmetric two-handed input actions and will allow us to adequately test our hypotheses. In addition, using this established two-handed task allows our study to extend the results reported by previous work [12].

Participants draw colored line segments between a set of twelve squares displayed on the screen (Fig. 1). The left hand holds and moves a ToolGlass widget which displays a $50 \%$ transparent four color palette. The ToolGlass always follows the left hand device; no button press is required. The right hand controls a cursor. At the start of the task, two 40x40 pixel squares appear on the screen. The starting square is colored black with a white border. The second (goal) square has one of the four colors (Red, Green, Blue, Yellow) found on the ToolGlass widget. The color segments on the ToolGlass widget are 50x50 pixels square, which allows it to overlap the target squares easily.

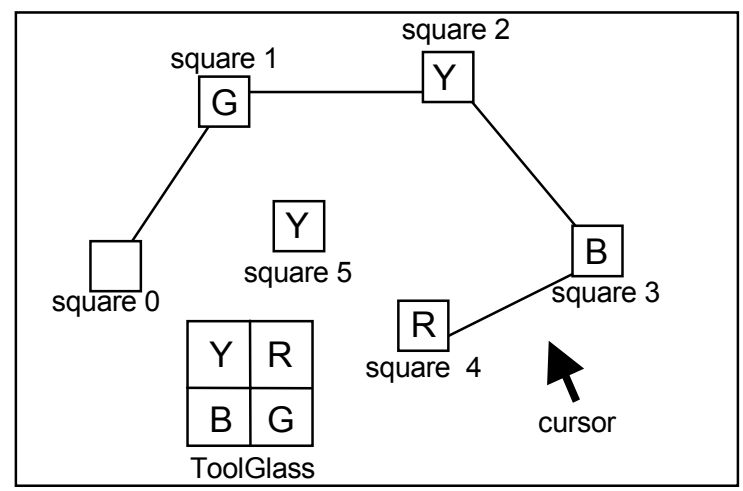

Fig. 1 Experimental task. Connect-the-dots task used in our experiment (and that of Kabbash [12]). $Y=$ Yellow, $\mathrm{R}=\mathrm{Red}, \mathrm{B}=\mathrm{Blue}, \mathrm{G}=\mathrm{Green}$. The text in this figure does not appear during the experiment.

Participants initiate line drawing by positioning the ToolGlass over the starting square, and using the main button on the right hand puck to position the cursor and click through the appropriate portion of the ToolGlass to match the color of the goal square. The right hand then draws a line to the goal square and indicates completion by releasing the button. A new square is then revealed and the participant repeats this process until all twelve squares have been connected. No two consecutive squares have the same color. The correct color must be selected to proceed. No errors are allowed, although we do record if this occurs.

\section{Apparatus}

A potential blunder in input device studies is to ignore the subtleties of the devices used, resulting in a simple device comparison that is of limited value. In our case, we want to explore the impact that varying kinesthetic reference frames may have on bimanual input without confounding the study with variations between input devices such as sampling rate, resolution, or physical form-factors.

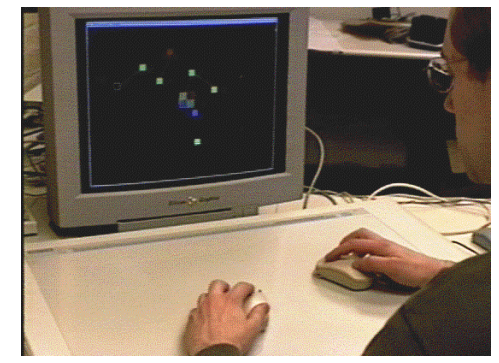

Fig. 2 Experiment set-up. Two pucks were used on a tablet placed in front of the display.

To achieve a carefully controlled analysis of our device factors, our strategy is to choose a very general input device (a Wacom tablet with two pucks) and use this hardware to emulate both absolute and relative input using identical hardware for each condition (Fig. 2). This allows us to keep the physical input hardware constant across our conditions (described in more detail below) and address a number of experimental questions without introducing uncontrolled confounds that might result, for example, from comparing a puck on a tablet to a regular mouse.

We use an 18x25 inch Wacom tablet for all experimental conditions. Commercially available Wacom tablets cannot sense two pucks on the same tablet; to address this we modified one of our two pucks such that it actually contained the sensor for a Wacom stylus. To emulate a clutching gesture for relative device mapping, we also modified the pucks so that each had a microswitch on the bottom of the device (which replaced one of the normal puck buttons). These microswitches detect when each puck is lifted from or placed back on the tablet surface.

We also use a simple 1:1 control-to-display mapping for all of our experimental conditions. This straightforward mapping allows us to study bimanual performance when basic motor-visual behavior is driving task performance, rather than increased levels of cognitive or other control issues that may arise with a more complex mapping.

The experiment ran on a Silicon Graphics Indigo2 Extreme workstation with a 21" display. The workstation ran in standalone mode, disconnected from all network traffic.

Although Wacom tablets similar to our apparatus are often used to implement two-handed interaction techniques, we cannot emphasize strongly enough that the present study uses the tablet as an experimental apparatus which enables us to vary the choice of kinesthetic reference frame in a controlled manner, resulting in a careful study of human bimanual performance issues. As such, our focus is on the human and not the particular input device which we employ as our testbed. 


\section{Experimental Conditions}

We implemented the following conditions to vary the kinesthetic reference frames (as shown in Figure 3).

Unified: Both hands operate in the same physical space, with a common invariant origin. This was achieved using an absolute tablet with two pucks sharing the input space. The left hand and right hand cursor positions were offset slightly by 1.5 inches to accommodate the devices and prevent them from bumping each other. This offset was constant.

Separated: Each hand operates in a separate physical space, each with its own invariant origin. This was achieved by using an absolute tablet divided into two halves. The left hand puck can only be used on the left side of the tablet, the right hand puck on the right side. Although each hand has its own space, there is a symmetry between the two spaces since their origins are offset only along the horizontal axis.

Relative: In this condition, each hand operates in its own separate space whose origin changes each time the device is clutched. We placed a cardboard template over the tablet. Two openings, approximately the size of a normal mouse pad, were cut in the template to provide a separate working area for each hand. Given the smaller working area, clutching of the pucks is allowed and indeed required, since the control-to-display ratio is the same as the other conditions. In our pilot studies, we found subjects have little or no need to ever clutch when a relative mapping uses the entire range of the tablet, resulting in behavior that is essentially identical to the Separated condition. Thus the template was used to emulate the practical constraint of device footprint. Note that we can calculate how much time the user spends clutching because we sense when each device is lifted or put down via our microswitches.

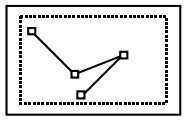

(a) Screen display

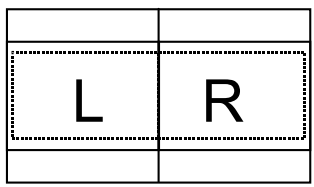

(c) Separated

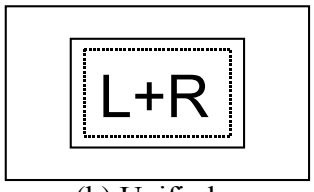

(b) Unified

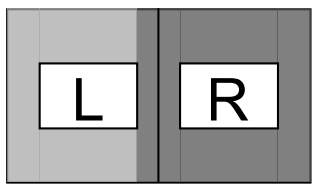

(d) Relative
Fig. 3 Mappings of the tablet to the screen. (a) Screen: The window on the screen is $11 \times 15$ inches. The dashed line shows the "active region" where dots are allowed to appear and measures 10x11". (b) Unified: Maps the "active region" directly to the central $10 \times 11$ " region of the tablet. (c) Separated: The "active region" is mapped to a $10 \times 11$ " area on either side of the tablet midline. (d) Relative: A cardboard template was fitted over the tablet. The cutouts were $6 \frac{1}{2} \times 10^{\prime \prime}$ and spaced $4 \frac{1}{4}$ " apart (centered on the tablet midline).
Note that other manipulations of the kinesthetic reference frames, such as left and right input spaces that differ by a scaling factor, or which are separated but offset both horizontally and vertically, are also possible. We chose not to explore these alternatives since they are not necessary to address our main hypotheses and they are also not representative of actual mappings that are used in practice.

We also varied visual feedback as follows:

Visual: The left hand ToolGlass and right hand cursor are always visible on the screen.

Nonvisual: The left hand ToolGlass is only shown when the virtual position of the ToolGlass is in the proximity of the right hand cursor position (twice the size of the ToolGlass), and only when not drawing a line. Once drawing starts, the ToolGlass disappears, and does not reappear until the user has moved it within proximity of the right hand cursor. Thus, when the ToolGlass is hidden, participants must rely on kinesthetic cues to move the ToolGlass within proximity of the right hand cursor.

Note that the Nonvisual condition does not remove all visual feedback because subjects can still see their hands and the tablet itself. While a total absence of visual feedback might be useful to further explore human performance issues, for this study we decided to use conditions which reflect typical computer usage, where users can indeed see their hands.

\section{Design and Procedure}

12 right handed participants took part in the study. The experiment was conducted in one sitting and lasted about one and a half hours per participant.

The visual feedback conditions were fully crossed with the kinesthetic reference frame conditions, yielding a withinsubjects factorial design with 6 experimental "kinesthetic/visual" conditions:

Unified-Visual (Uv) Unified-Nonvisual (Un)

Separated-Visual (Sv) Separated-Nonvisual (Sn)

Relative-Visual (Rv) Relative-Nonvisual (Rn)

These conditions were counterbalanced as follows:

\begin{tabular}{|c|l|c|c|}
\hline Ss & $\begin{array}{l}\text { Group 1: } \\
\text { Visual first }\end{array}$ & Ss & $\begin{array}{l}\text { Group 2: } \\
\text { Nonvisual first }\end{array}$ \\
\hline 1 & U, S, R & 7 & U, S, R \\
\hline 2 & U, R, S & 8 & U, R, S \\
\hline 3 & S, U, R & 9 & S, U, R \\
\hline 4 & S, R, U & 10 & S, R, U \\
\hline 5 & R, U, S & 11 & R, U, S \\
\hline 6 & R, S, U & 12 & R, S, U \\
\hline
\end{tabular}

Fig. 4 Experimental design. Group 1 performed the Visual condition first, followed immediately by the Nonvisual condition: i.e., Participant 1 performed Uv, then Un, then Sv, then $\mathrm{Sn}$, then Rv, then Rn. Group 2 performed the Nonvisual conditions first. 
For each condition, participants performed 5 blocks of trials. Each block consisted of 2 sets of 12 squares to connect. One set had the constraint that the squares appeared at an Euclidean distance of 200 pixels away from the previous square. In the second set, the squares appeared at a distance of 500 pixels apart. In both sets, the size of the squares was kept constant. The location of the squares between every set of trials was randomized, with the constraint that drawn lines would not cross each other. The order of appearance of the 200 pixel set and the 500 pixel set was randomized within each block.

During pilot testing, we also included sets with 300 and 400 pixel distances and varied the size of the squares, as is typically done in target selection experiments. However, we found that target size had no effect on the relative performance of the six experimental conditions, and performance varied linearly over the range of distances. Using a single target size, and just the 200 and 500 pixel distances, allows us to measure performance for both extremes while keeping the experiment duration manageable.

Prior to starting each kinesthetic /visual condition, participants were given three warm-up sets of squares to familiarize themselves with the condition. They were allowed breaks between each set of squares. The experiment consisted of a total of 720 sets of squares to connect:

12 participants $X$

6 kinesthetic/visual conditions $x$

5 blocks per condition $x$

2 sets of 12 squares per condition

$=720$ sets of squares to connect

\section{Hypotheses}

We expect to find the following effects in our experimental data:

H1. Experimental manipulation of the kinesthetic reference frames will not have a significant impact on performance when visual feedback is present. That is, conditions where visual feedback is present $(\mathrm{Uv}, \mathrm{Sv}$, and $\mathrm{Rv})$ will not differ significantly.

H2. In the absence of visual feedback, mappings which separate the kinesthetic reference frames for each hand will result in significantly slower performance (i.e., the means of our experimental conditions will be in the order $\mathrm{Un}<\mathrm{Sn}$ $<\mathrm{Rn}$ ), but a mapping where the right and left hands share a unified kinesthetic reference frame will not (i.e., $\mathrm{Un}=\mathrm{Uv}$ ).

\section{Results}

The overall means for our experimental conditions are shown in Figure 5. We report two results for the Relative conditions: Relative with clutching is the raw completion time data we collected. Relative is completion time with clutching removed. This was calculated by subtracting out all time where either the right or left hand was engaged in a clutching motion. It is possible for one hand to clutch while the other hand is still in motion. We decided to always subtract out this time to produce a conservative (optimistic) measure of Relative performance. All of our subsequent data analyses use completion time for Relative with clutching removed.

We removed 11 outlier trials (more than 3 standard deviations from the mean) from the 720 experimental trials (approximately 1\%). No more than $4 \%$ of trials in any particular condition were removed.

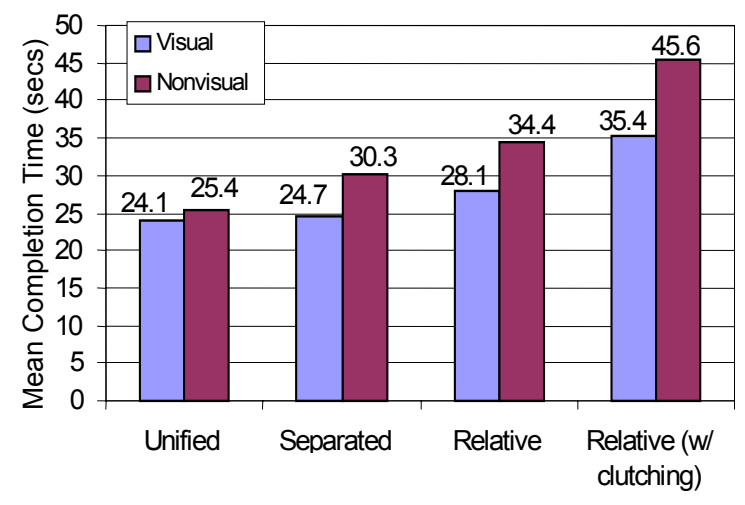

Fig. 5 Summary of results.

We performed a $3 \times 2 \times 5 \times 2$ repeated measures analysis of variance on the within-subject factors of Kinesthetic Frame of reference ( $\mathrm{KF}=$ Unified, Separated, Relative), Visual Feedback ( $\mathrm{VF}=$ Visual, Nonvisual), Block (5), and Distance (200, 500 pixels), with task completion time as the dependent variable. We also analyzed the between-subject factors of Group (Visual first, Nonvisual first) and Order of conditions. Significant effects are summarized in Table 2.

\begin{tabular}{|l|l|l|}
\hline Factor & $F_{\text {value }}$ & p \\
\hline KF & $\mathrm{F}_{(2,10)}=64.87$ & $\mathrm{p}<.0001$ \\
\hline VF & $\mathrm{F}_{(1,5)}=59.00$ & $\mathrm{p}<.001$ \\
\hline Distance & $\mathrm{F}_{(1,5)}=360.97$ & $\mathrm{p}<.0001$ \\
\hline KF X VF & $\mathrm{F}_{(2,10)}=14.85$ & $\mathrm{p}<.001$ \\
\hline KF X Distance & $\mathrm{F}_{(2,10)}=27.96$ & $\mathrm{p}<.0001$ \\
\hline KF X Block & $\mathrm{F}_{(8,40)}=3.04$ & $\mathrm{p}<.01$ \\
\hline KF X Order & $\mathrm{F}_{(2,10)}=5.86$ & $\mathrm{p}<0.005$ \\
\hline VF X Distance & $\mathrm{F}_{(1,5)}=39.19$ & $\mathrm{p}<.002$ \\
\hline VF X Group & $\mathrm{F}_{(1,5)}=13.13$ & $\mathrm{p}<.02$ \\
\hline KF X VF X Distance & $\mathrm{F}_{(2,10)}=17.20$ & $\mathrm{p}<.001$ \\
\hline KF X VF X Block & $\mathrm{F}_{(8,40)}=2.40$ & $\mathrm{p}<.05$ \\
\hline KF X Block X Dist. & $\mathrm{F}_{(8,40)}=2.25$ & $\mathrm{p}<.05$ \\
\hline
\end{tabular}

Fig. 6 Significant effects.

There was a main effect for the KF and VF factors, as well as a KF $\mathrm{x}$ VF interaction. Pairwise means comparison tests showed that the Uv, Sv, and Rv conditions did not differ significantly. This supports hypotheses H1. Pairwise means comparison also revealed that the $\mathrm{Rn}$ and $\mathrm{Sn}$ conditions differed significantly from the other means, while Un and Uv conditions did not differ from one another, thus confirming hypothesis $\mathrm{H} 2$. 
As expected, the sets with longer Euclidean distance between squares took more time to complete. The KF $\mathrm{x}$ Distance, VF $x$ Distance, and KF $x$ VF $x$ Distance interactions indicate that certain $\mathrm{KF}$ and $\mathrm{VF}$ conditions affected performance for the two Distance conditions by different extents. Pairwise means comparisons indicate no difference between all the 200 pixel Distance conditions except for the $\mathrm{Rn}$ condition. For the 500 pixel Distance condition, the $\mathrm{Sn}, \mathrm{Rv}$, and $\mathrm{Rn}$ conditions differed from the others. These results indicate that separated kinesthetic reference frames and the absence of visual feedback is detrimental regardless of the amount of movement required to complete the task ( $\mathrm{Rn}$ condition, both 200 and 500 pixels distance). Furthermore, in the absence of visual feedback, when the kinesthetic reference frame of each hand is separated but the workspace origins remain constant, performance is reduced only as the amount of movement required increases ( $\mathrm{Sn}$ condition, 500 pixels distance). Finally, use of the Relative mapping even in the presence of visual feedback can slightly affect task performance if the amount of movement required is large ( $\mathrm{Rv}$ condition, 500 pixels distance); it is possible that with the larger movement distance, the likelihood of clutching becoming necessary was higher, and such interruptions were sufficient to significantly reduce performance.

The above paragraphs account for the dominant effects. The other, less dominant, but still statistically significant effects are as follows:

- Inspection of the data indicate that the KF $\mathrm{x}$ Block interaction resulted from little or no learning occurring in the Unified and Separated conditions (as evident from the lack of a main effect for Block), but a slight learning effect existed for the Relative condition.

- Inspection of the means suggest that the KF $\mathrm{x}$ Order interaction is possibly due to a slight negative training effect when the Relative conditions are performed last.

- As may be expected, participants took a little longer to learn the task when they did the Nonvisual conditions first. This accounts for the VF x Group interaction. The $\mathrm{KF} \times \mathrm{VF} \times$ Block interaction is due to slight learning occurring in the $\mathrm{Rn}$ condition. Similarly the $\mathrm{KF} \mathrm{x}$ Block $x$ Distance interaction is due to a larger extent of learning occurring in the $\mathrm{Rn}, 500$ pixel condition.

\section{DISCUSSION}

The results in support of our experimental hypotheses suggest three high-level findings:

First, our results suggest that visual reference in feedback on the screen is sufficient for Guiard's reference principle to apply; hence absolute spatial kinesthetic reference (a direct correspondence between the physical separation of the hands versus the position of the hands as sensed by input devices) is not an essential property of the reference principle. This is supported by our results which show that, despite the varying kinesthetic reference frames, performance is very similar across the Visual conditions (once clutching time is accounted for in the Relative condition). The remarkably small difference between the Visual and Nonvisual conditions for the Unified tablet also shows that Guiard's reference principle holds without visual feedback if body-relative kinesthetic cues are available, and thus in this sense visual feedback is not an essential property of the reference principle either.

If we had observed robust differences across the Uv, Sv, and $\mathrm{Rv}$ conditions over both short and long distances (that is, if $\mathrm{H} 1 \mathrm{did}$ not hold), this would have suggested a possible dependency of the Guiard reference principle on the workspace and origin of the hands. But we found little evidence to support this conclusion, and our results thus suggest that the Guiard reference principle is not dependent on the two hands operating in a unified workspace with a common origin 2 . Of course, the lack of evidence does not prove that no difference can exist, but it is certainly very suggestive that the mapping of the kinesthetic reference frames, at least among the common such mappings explored by our experiment, is not a significant factor when visual feedback is present.

Second, when both visual and kinesthetic feedback are available, vision is the dominant feedback channel. Even if kinesthetic feedback is poor, visual feedback can guide performance without significant detriment. The feedback loop is apparently not any slower when it depends on visual feedback (again supported by the similarity of the Visual conditions, within the statistical sensitivity of our experiment). However, once visual feedback is removed, separated kinesthetic reference frames for each hand can potentially have a severe penalty for performance (supported by the significant reduction in performance for the Separated-Nonvisual condition and especially the Relative-Nonvisual condition).

Third, this evidence suggests that body-relative kinesthetic cues, such as those afforded by our Unified condition, are sufficient to guide motion in the Nonvisual case and thus can reduce the user's dependence on visual feedback to guide bimanual actions. However, when the origin of the kinesthetic reference frames changes frequently, the user becomes increasingly dependent on visual feedback, especially as the magnitude of motion increases.

\section{Implications for Devices \& Interaction}

These findings have significant implications for the design of input devices and two-handed interaction techniques. In short, we see these findings as good news in the sense that two-handed input is fairly robust with respect to various mappings of the input space, meaning that even devices that cannot sense the physical separation of the two hands in a common reference frame can still provide effective twohanded input as long as the user's visual attention can be fully focused on the task. However, if a high level of visual

\footnotetext{
${ }^{2}$ We did find a significant KF X VF X Distance interaction because the $\mathrm{Rv}$ condition at 500 pixels distance differed significantly from the other visual conditions.
} 
diversion is required for a task or interaction technique (as simulated by our Nonvisual conditions) emphasis must be given to a system with a unified kinesthetic reference frame, such as that afforded by the Wacom tablet.

This also means that some of the observed limitations (e.g. Zeleznik et. al. [26]) of current mice for two-handed input are essentially technological limitations (caused, for example, by the limited precision of existing roller-ball sensing mechanisms) rather than fundamental limitations of human performance. For example, if one postulates an improved mouse technology that can reasonably track absolute position, it would be possible to use one mouse in each hand (perhaps on either side of a keyboard) as a separate "tablet." Our experimental data suggests that such hypothetical devices would yield performance similar to our Separated condition if there was enough room to use them without clutching, or similar to the Relative condition if fairly frequent clutching occurs. It is also important to keep in mind that a two-handed input technique, even using the limited capabilities of current mice, can still significantly outperform one-handed input techniques [2].

Note that one cannot conclude from our study that "relative devices are bad." Relative devices can have a number of properties such as small footprint, low cost, and a comfortable range of motion, which compel their use in many situations. Indeed, our results suggest that relative devices have few fundamental problems with respect to human performance if visual attention is focused on the task.

The present experiment uses a simple one-to-one mapping of the input space to the display space. Many computer tasks, such as virtual camera control [2], can benefit from or indeed require an abstract mapping from input to display. The influence of kinesthetic reference frames may be diminished for such tasks since the user must rely on vision to guide hand movement. Our experimental data suggests that such mappings might indeed be useful in two-handed input since we found that the dominant influence of visual feedback is sufficient to mask significant deficiencies in kinesthetic reference.

\section{Some Unresolved Issues}

Our experimental task was relatively simple, yet cognitive load and task complexity may also influence two-handed input performance. Since there is a greater motor load with two-handed input, increasing cognitive load or task complexity may affect two-handed input more than onehanded input. This must be balanced against the expected benefits of two-handed interaction, which of itself can potentially yield cognitive benefits [15]. One way to explore this issue might be an experiment which introduces a secondary task to increase cognitive load.

The present study also does not address two-handed tasks which may require a higher degree of inter-hand coordination. The ToolGlass task [12] essentially requires coordination with respect to visual targets on the screen. In other tasks, such as two-handed map manipulation [7] the gesture of bringing one's hands together is an important skill. Such movements seem characteristic of symmetric bimanual action, as opposed to the asymmetric action we have focused on so far. This raises the issue of whether symmetric and asymmetric bimanual gestures are fundamentally different, or whether a generalization of Guiard's principles could apply to both cases. In the workflow of some two-handed input systems (e.g. Kurtenbach et. al. [14]) one can observe fluid transitions between asymmetry and symmetry, such as using a ToolGlass selection to initiate a symmetric two-handed stretching task. With the exception of Leganchuk et. al. [15], there is little experimental data on symmetric twohanded input tasks, suggesting this is an area ripe for further experimental study.

\section{CONCLUSION \& FUTURE WORK}

We have presented experimental work that explores the interactions between mappings of the input work space and the availability of visual feedback for two-handed interaction. Our experimental data suggests that the Guiard reference principle applies to visual as well as kinesthetic reference. Vision is also clearly the dominant feedback channel as it can overcome significant limitations in kinesthetic reference afforded by indirect mappings of the input work space. We have discussed the relevance of these findings for input devices and interaction techniques. We have also brought up some issues that remain unresolved by the current experiment.

In addition to the unresolved issues above, several related issues could be explored by future work. We have measured two-handed input performance in terms of task completion time, but one could extend Zhai and Milgram's metric of coordination [27] to bimanual input and perhaps get a deeper understanding of the effects of varying the kinesthetic reference frames. One could also vary the kinesthetic reference frames more systematically than we have in the current experiment, for example by looking at conditions with scale non-correspondence (i.e. a constant, but non-1:1 control-to-display mapping). Nonetheless, our straightforward experiment and outcome measures were sufficient to explore a number of previously unresolved issues in two-handed input.

\section{ACKNOWLEDGEMENTS}

We thank Gordon Kurtenbach, George Fitzmaurice, and Russell Owen for valuable discussions and assistance during the course of this work. We also thank Alias|wavefront and Microsoft for supporting this collaborative research study.

\section{REFERENCES}

1. Annett, J., Annett, M., Hudson, P., Turner, A., "The Control of Movement in the Preferred and NonPreferred Hands," Quarterly Journal of Experimental Psychology, 31: p. 641-652, 1979. 
2. Balakrishnan, R., Kurtenbach, G., "Exploring Bimanual Camera Control and Object Manipulation in 3D Graphics Interfaces," CHI'99 Conference on Human Factors in Computing Systems, 1999, 56-63.

3. Bier, E.A., "Snap-Dragging In Three Dimensions," Computer Graphics (Proc. 1990 Symposium on Interactive 3D Graphics), 24 (2): p. 193-204, 1990.

4. Buxton, W., Myers, B., "A Study in Two-Handed Input," Proceedings of CHI'86: ACM Conference On Human Factors in Computing Systems, 1986, 321-326.

5. Guiard, Y., "Asymmetric Division of Labor in Human Skilled Bimanual Action: The Kinematic Chain as a Model," The Journal of Motor Behavior, 19 (4): p. 486-517, 1987.

6. Guiard, Y., T. Ferrand, T., "Asymmetry in Bimanual Skills," in Manual asymmetries in motor performance, Elliott and Roy, Editors. 1995, CRC Press: Boca Raton, FL.

7. Hinckley, K., Czerwinski, M., Sinclair, M., "Interaction and Modeling Techniques for Desktop Two-Handed Input," Proceedings of the ACM UIST'98 Symposium on User Interface Software and Technology, 1998, 49-58.

8. Hinckley, K., Pausch, R., Proffitt, D., Kassell, N., "Attention and Visual Feedback: The Bimanual Frameof-Reference," ACM/SIGGRAPH Symposium on Interactive 3D Graphics, 1997, 121-126.

9. Hinckley, K., Pausch, R., Proffitt, D., Kassell, N., "Two-Handed Virtual Manipulation," ACM Transactions on Computer-Human Interaction, 5 (3 (Sept.)): p. 260-302, 1998.

10. Hinckley, K., Pausch, R., Proffitt, D., Patten, J., Kassell, N., "Cooperative Bimanual Action," Proceedings of CHI'97: ACM Conference on Human Factors in Computing Systems, 1997, 27-34.

11. Hinckley, K., Tullio, J., Pausch, R., Proffitt, D., Kassell, N., "Usability Analysis of 3D Rotation Techniques," Proceedings of the ACM UIST'97 Symposium on User Interface Software and Technology, 1997, 1-10.

12. Kabbash, P., Buxton, W., Sellen, A., "Two-handed input in a compound task," Proceedings of CHI'94: ACM Conference on Human Factors in Computing Systems, 1994, 417-423.

13. Kelso, J., Southard, D., Goodman, D., "On the Coordination of Two-Handed Movements," Journal of Experimental Psychology: Human Perception and Performance, 5 (2): p. 229-238, 1979.

14. Kurtenbach, G., Fitzmaurice, G., Baudel, T., Buxton, B., "The Design of a GUI Paradigm based on Tablets, Two-hands, and Transparency," Proceedings of
CHI'97: ACM Conference on Human Factors in Computing Systems, 1997, 35-42.

15. Leganchuk, A., Zhai, S., Buxton, W., "Manual and Cognitive Benefits of Two-Handed Input: An Experimental Study," ACM Transactions on CHI, 5 (4): p. 326-359, 1999.

16. Marteniuk, R., MacKenzie, C., Baba, D., "Bimanual movement control: Information Processing and interaction effects," Quarterly Journal of Experimental Psychology, 36A: p. 335-365, 1984.

17. Mine, M., Brooks, F., Sequin, C., "Moving Objects in Space: Expoiting Proprioception in VirtualEnvironment Interaction," SIGGRAPH'97, 1997, .

18. Peters, M., "Constraints in the performance of bimanual tasks and their expression in unskilled and skilled subjects," Quarterly Journal of Experimental Psychology, 37A: p. 171-196, 1985.

19. Preilowski, B., "Possible Contribution of the Anterior Forebrain Commissures to Bilateral Motor Coordination," Neuropsychologia, 10: p. 267-277, 1972.

20. Preilowski, B., "Intermanual transfer, interhemispheric interaction, and handedness in man and monkeys," in Brain Circuits \& Functions of the Mind: Essays in Honor of Roger W. Sperry, C. Trevarthen, Editor. 1990, Cambridge Univ. Press.

21. Provins, K., Glencross, D., "Handwriting, typewriting and handedness," Quarterly Journal of Experimental Psychology, 20: p. 282-289, 1968.

22. Sellen, A., Kurtenbach, G., Buxton, W., "The Prevention of Mode Errors through Sensory Feedback," Human Computer Interaction, 7 (2): p. 141-164, 1992.

23. Wickens, C., "Chapter 3: Attention in Perception and Display Space," in Engineering Psychology and Human Performance. 1992, HarperCollins: New York. p. $74-115$.

24. Wing, A., "Timing and co-ordination of repetitive bimanual movements," Quarterly Journal of Experimental Psychology, 34A: p. 339-348, 1982.

25. Wolff, P., Hurwitz, I., Moss, H., "Serial organization of motor skills in left- and right-handed adults," Neuropsychologia, 15: p. 539-546, 1977.

26. Zeleznik, R., Forsberg, A., Strauss, P., "Two pointer input for 3D interaction.," ACM/SIGGRAPH Symposium on Interactive 3D Graphics, 1997, 115120.

27. Zhai, S., Milgram, P., "Quantifying Coordination in Multiple DOF Movement and Its Application to Evaluating 6 DOF Input Devices," CHI'98, 1998, 320327. 